University of Texas at El Paso

ScholarWorks@UTEP

$12-2017$

\title{
Beyond Integration: A Symmetry-Based Approach to Reaching Stationarity in Economic Time Series
}

Songsak Sriboonchitta

Chiang Mai University, songsakecon@gmail.com

Olga Kosheleva

The University of Texas at El Paso, olgak@utep.edu

Vladik Kreinovich

The University of Texas at El Paso, vladik@utep.edu

Follow this and additional works at: https://scholarworks.utep.edu/cs_techrep

Part of the Computer Sciences Commons

Comments:

Technical Report: UTEP-CS-17-97

To appear in: Olga Kosheleva, Sergey Shary, Gang Xiang, and Roman Zapatrin (eds.), Beyond Traditional Probabilistic Data Processing Techniques: Interval, Fuzzy, etc. Methods and Their Applications, Springer, Cham, Switzerland, 2018.

\section{Recommended Citation}

Sriboonchitta, Songsak; Kosheleva, Olga; and Kreinovich, Vladik, "Beyond Integration: A Symmetry-Based Approach to Reaching Stationarity in Economic Time Series" (2017). Departmental Technical Reports (CS). 1190.

https://scholarworks.utep.edu/cs_techrep/1190

This Article is brought to you for free and open access by the Computer Science at ScholarWorks@UTEP. It has been accepted for inclusion in Departmental Technical Reports (CS) by an authorized administrator of ScholarWorks@UTEP. For more information, please contact Iweber@utep.edu. 


\title{
Beyond Integration: A Symmetry-Based Approach to Reaching Stationarity in Economic Time Series
}

Songsak Sriboonchitta, Olga Kosheleva, and Vladik Kreinovich

\begin{abstract}
Many efficient data processing techniques assume that the corresponding process is stationary. However, in areas like economics, most processes are not stationery: with the exception of stagnation periods, economies usually grow. A known way to apply stationarity-based methods to such processes - integration - is based on the fact that often, while the process itself is not stationary, its first or second differences are stationary. This idea works when the trend polynomially depends on time. In practice, the trend is usually non-polynomial: it is often exponentially growing, with cycles added. In this paper, we shod how integration techniques can be expanded to such trends.
\end{abstract}

\section{Formulation of the Problem}

Need to reach stationarity. Many efficient statistical techniques are based on the assumption that the corresponding random process is stationary, i.e., that its characteristics do not change in time.

In many real-life applications, stationarity is indeed a reasonable assumption. However, in economics, stationarity means stagnation. This may have been true in middle ages, but definitely not now - all over the world, economies are growing. However, very few statistical tools exist for such non-stationary processes as economic growth.

So, since we cannot directly apply stationarity-based techniques to most economic variables, it is desirable to come up with ideas on how to apply such tech-

Songsak Sriboonchitta

Faculty of Economics, Chiang Mai University, Thailand

e-mail: songsakecon@gmail.com

Olga Kosheleva and Vladik Kreinovich

University of Texas at El Paso, El Paso, TX 79968, USA

e-mail: olgak@utep.edu,vladik@utep.edu 
niques indirectly, i.e., how to reach stationarity based on the original non-stationary process $x_{t}$.

Integration: a widely used approach to reach stationarity. The economy-related variables $x_{t}$ - such as the prices or stock market index - usually contain a slowly changing trend $T_{t}$, on top of which we have random fluctuations $f_{t}$ :

$$
x_{t}=T_{t}+f_{t} .
$$

The fluctuations usually are stationary - at least for a certain reasonable period of time, what is non-stationary is the trend $T_{t}$.

The simplest possible trend is when we have a linear growth $T_{t}=a+b \cdot t$. In this case,

$$
x_{t}=a+b \cdot t+f_{t} .
$$

In this case, as one can easily see, first differences $\Delta x_{t} \stackrel{\text { def }}{=} x_{t}-x_{t-1}$ form a stationary process; namely,

$$
\Delta x_{t}=x_{t}-x_{t-1}=\left(a+b \cdot t+f_{t}\right)-\left(a+b \cdot(t-1)+f_{t-1}\right)=b+f_{t}-f_{t-1} .
$$

Here, $b$ is a constant, and since $f_{t}$ is stationary, the difference $f_{t}-f_{t-1}$ is stationary as well. So, while the original random process is not stationary, we can apply stationarity-based techniques to the differences $\Delta x_{t}$. This procedure is known as integration of order 1; see, e.g., [1, 3].

The procedure of first-order co-integration is based on the assumption that the trend is uniformly increasing. In practice, the trend may accelerate or decelerate. To describe such acceleration or deceleration, we can - similarly to how we take into account acceleration or deceleration in mechanics - add terms which ate quadratic in time to our description of the trend. In this case, $T_{t}=a+b \cdot t+c \cdot t^{2}$ and thus,

$$
x_{t}=a+b \cdot t+c \cdot t^{2}+f_{t} .
$$

For such more complicated trend, first differences are not longer stationary:

$$
\begin{gathered}
\Delta x_{t}=x_{t}-x_{t-1}= \\
\left(a+b \cdot t+c \cdot t^{2}+f_{t}\right)-\left(a+b \cdot(t-1)+c \cdot(t-1)^{2}+f_{t-1}\right)= \\
b+2 c \cdot t-c+f_{t}-f_{t-1} .
\end{gathered}
$$

Good news, however, is that the form (5) is exactly the form (2), in which the new trend is linear. Thus, we can use the same idea to reach stationarity: namely, we can take the first difference of $\Delta x_{t}$ and consider the new times series $\Delta^{2} x_{t}=\Delta\left(\Delta x_{t}\right)=$ $\Delta x_{t}-\Delta x_{t-1}$. For this time series,

$$
\begin{gathered}
\Delta^{2} x_{t}=\Delta x_{t}-\Delta x_{t-1}= \\
\left(b+2 c \cdot t-c+f_{t}-f_{t-1}\right)-\left(b+2 c \cdot(t-1)-c+f_{t-1}-f_{t-2}\right)=
\end{gathered}
$$




$$
2 c+f_{t}-2 f_{t-1}+f_{t-2} \text {. }
$$

The resulting time series is clearly a stationary process. This is known as integration of order 2.

If we want to make our model even more accurate and take into account that the acceleration also changes with time, we can add terms cubic in time to the trend, in which case the time series $\Delta^{3} x_{t} \stackrel{\text { def }}{=} \Delta\left(\Delta^{2} x_{y}\right)=\Delta^{2} x_{t}-\Delta^{2} x_{t-1}$ are stationary, etc.

This has become a standard procedure in analyzing economic data: first, we check if after the integration of appropriate order, we get a stationary process, and then we apply stationarity-based statistical methods to the resulting stationary process.

Need to go beyond integration. Integration works well when the trend is a polynomial function of time. From the mathematical viewpoint, on a reasonably short time interval, any smooth dependence $T_{t}$ can be expanded in Taylor series and thus, well approximated by a polynomial. So, locally, integration works well.

However, in economics, we are often interested in long-term trends. And for long-term trends, polynomial approximation does not always work well. Let us give two simple examples.

An ideal regime of an economics is a growth at constant rate, when the GDP in the next year is larger that the GDP of the previous year by the same factor $1+q$. In this case, the growth is described by a geometric progression $T_{t}=T_{0} \cdot(1+q)^{t}$. This is a simple and natural function - but it is not a polynomial. As a result, no matter how many times we apply the finite difference operator $\Delta$, we will never reach a stationary process.

Ideally, we should have a consistent growth, but in reality, on top of this growth, we also have business cycles: periods of faster growth are followed by periods of slower growth, then faster growth resumes, etc. A simple description of such a cycle is a sinusoid, when $T_{t}=T_{0} \cdot(1+q)^{t}+A \cdot \sin (\omega \cdot t+\varphi)$. A more adequate description is when we take into account that the size of the sinusoidal fluctuations is not constant, but growth when the economy's level grows, i.e., that

$$
T_{t}=T_{0} \cdot(1+q)^{t}+A \cdot(1+q)^{t} \cdot \sin (\omega \cdot t+\varphi) .
$$

It is therefore desirable to come up with techniques that would enable us to reach stationary for such non-polynomial trends as well.

What we do in this paper. In this paper, we explain, in the most general setting, how to reach stationarity.

\section{Analysis of the Problem}

Let us describe the class of possible trends $T_{t}$. To come up with such a general scheme, let us describe the class of possible time series $T_{t}$ describing trend. 
The class of possible trends must not change if we change a measuring unit. The numerical value of each economic quantity depends on the unit that we use to measure it. For example, if we measure the Thailand GDP in Baht, we get a different number than if we measure it in US dollars. In general, if we replace the original measuring unit with a new unit which is $\lambda$ times smaller than the original one, all numerical values get multiplied by this value $\lambda$. So, instead of the original time series $T_{t}$, we get a new time series $\lambda \cdot T_{t}$.

The new time series describes the exact same phenomenon as the original one the only difference is that it uses different measuring units. So, if the original time series $T_{t}$ was reasonable, the new time series $\lambda \cdot T_{t}$ should be reasonable as well.

In mathematical terms, the class $C$ of reasonable time series should be closed under multiplication by a constant.

The class of possible trends should be closed under addition. Many economic characteristics are obtained by adding up several others. For example:

- the GDP of a country is equal to the sum of GDPs of the region,

- a stock market index is equal to a linear combination of the stock prices of different stocks, etc.

Thus, if $T_{t}$ and $T_{t}^{\prime}$ are possible trends, it is reasonable to assume that their sum $T_{t}+T_{t}^{\prime}$ is a possible trend as well.

In mathematical terms, this means that the class $C$ of reasonable time series should be closed under addition.

First conclusion: the class of possible trends should form a linear space. Since the class $C$ is closed under addition and under multiplication by a constant, with each set $T_{t}, T_{t}^{\prime}, T_{t}^{\prime \prime}, \ldots$, and for all possible values $c, c^{\prime}, c^{\prime \prime}, \ldots$, the linear combination $c \cdot T_{t}+c^{\prime} \cdot T_{t}^{\prime}+c^{\prime \prime} \cdot T_{t}^{\prime \prime}+\ldots$ should also belong to this class.

In mathematical terms, this means that the class $C$ of reasonable time series should form a linear space.

The class of possible trends should be closed under time shift. From the economic viewpoint, there is nothing special about any year, be it year 0 in the Western calendar or year 0 in Thai calendar. If a time series $T_{t}$ is possible, then a similar time series $T_{t}^{\prime} \stackrel{\text { def }}{=} T_{t+t_{0}}$ but starting a year earlier (when $t_{0}=1$ ) or a year later (when $t_{0}=-1$ ) should also be possible.

In mathematical terms, this means that the class of possible trends should be closed under time shifts $T_{t} \rightarrow T_{t}^{\prime}=T_{t+t_{0}}$.

\section{Examples.}

- The class of all polynomials of a given order is clearly closed under the shift.

- The class of geometric progressions $T_{t}=T_{0} \cdot(1+q)^{t}$ is also shift-invariant: namely,

$$
T_{t+t_{0}}=t_{0} \cdot(1+q)^{t+t_{0}}=T_{0} \cdot(1+q)^{t_{0}} \cdot(1+q)^{t}=T_{0}^{\prime} \cdot(1+q)^{t},
$$

where $T_{0}^{\prime} \stackrel{\text { def }}{=} T_{0} \cdot(1+q)^{q_{0}}$. 
- Simple cycles $A \cdot(\sin (\omega \cdot t+\varphi)$ can be equivalently represented as

$$
c_{1} \cdot \sin (\omega \cdot t)+c_{2} \cdot \cos (\omega \cdot t) .
$$

By using the formulas for the sine and cosine of the sum, one can easily check that this class is also shift-invariant.

- Similarly, one can prove that the above classes

$$
T_{t}=T_{0} \cdot(1+q)^{t}+A \cdot \sin (\omega \cdot t+\varphi)
$$

and

$$
T_{t}=T_{0} \cdot(1+q)^{t}+A \cdot(1+q)^{t} \cdot \sin (\omega \cdot t+\varphi)
$$

are shift-invariant.

The class of possible trends should depend on finitely many parameters. The last reasonable requirement is that it should be possible to uniquely determine a possible trend by using only finitely many parameters - and ideally, not a very large number of parameters.

Indeed, our goal is to determine the trend based on the observations. Each observation leads to one equation for determining the parameters. Thus, to determine all the parameters, we have a system of finitely many equations - as many equations as we have observations.

In general, to be able to solve a system of equations, we need to have at least as many equations as there are unknowns - otherwise, we will not be able to uniquely determine all the unknowns. Thus, to be able to - at least in principle - determine the trend based on the observations, we need to make sure that the number of parameters describing the trend is finite - less than or equal to the number of possible observations.

We know that the class $C$ of all possible trends is a linear space. It is known that in a linear space, we can always select the maximum set of linearly independent elements - known as basis - so that each element of a linear space can be described as a linear combination of elements from the cases. Thus, to uniquely determine an element of a linear space, we need to describe as many parameters as there are elements in the basis - this number is known as the dimension of the linear space.

So, we can conclude that the linear space $C$ of all possible trends is finitedimensional.

Now, we are ready to describe our main result.

\section{A General Approach to Reaching Stationarity}

Towards a matrix formulation. Since the linear space $C$ of all possible trends is finite-dimensional, it has a basis $e_{1, t}, \ldots, e_{d, t}$ where $d$ is the dimension of this space. Thus. every possible trend $T_{t} \in C$ can be represented as a linear combination of the 
basis elements:

$$
T_{t}=\sum_{j=1}^{d} c_{j} \cdot e_{j, t} .
$$

In particular, each of the basic sequences $e_{i, t}$ is possible. Since the class of possible sequences is invariant under shift, the shifted sequence $e_{i, t+1}$ is also possible. Since this sequence is possible, it can be represented in the form (7) for appropriate coefficients:

$$
e_{i, t+1}=\sum_{j=1}^{n} c_{i, j} \cdot e_{j, t} .
$$

This equality can be naturally described in matrix terms: namely, if, for each moment $t$, we consider the vector $E_{t}$ consisting of the elements $e_{1, t}, \ldots, e_{d, t}$, then the equation (8) takes the form

$$
E_{t+1}=C E_{t},
$$

where $C$ is a $d \times d$ matrix with coefficients $c_{i, j}$, and $C E_{t}$ means multiplying the matrix $C$ and the vector $E_{t}$. In these terms, the formula (7) takes the form

$$
T_{t}=c^{\mathrm{T}} E_{t}
$$

where $c$ is the vector consisting of the coefficients $c_{1}, \ldots, c_{d}$.

From (9), we can conclude that $E_{t+2}=C E_{t+1}=C\left(C E_{t}\right)=C^{2} E_{t}$, and similarly, that

$$
E_{t+t_{0}}=C^{t_{0}} E_{t}
$$

Towards the resulting formula for $T_{t}$. It is known - this statement is known as the Cayley-Hamilton theorem (see, e.g., [2]) - that each matrix $C$ satisfies a polynomial equation: namely, if we consider its characteristic polynomial

$$
\chi(\lambda) \stackrel{\text { def }}{=} \operatorname{det}(C-\lambda)=a_{n} \lambda^{n}+a_{n-1} \lambda^{n-1}+\ldots+a_{1} \cdot \lambda+a_{0},
$$

and then plug in the matrix $C$ into this polynomial, we get 0 :

$$
a_{n} \cdot C^{n}+a_{n-1} \cdot C^{n-1}+\ldots+a_{1} \cdot C+a_{0} \cdot I=0,
$$

where $I$ denotes a unit matrix, with 1 s on diagonal and 0s elsewhere.

Multiplying both sides of (12) by $E_{t}$, we get

$$
a_{n} \cdot C^{n} E_{t}+a_{n-1} \cdot C^{n-1} E_{t}+\ldots+a_{1} \cdot C E_{t}+a_{0} \cdot E_{t}=0,
$$

i.e., due to (11):

$$
a_{n} \cdot E_{t+n}+a_{n-1} \cdot E_{t+(n-1)}+\ldots+a_{1} \cdot E_{T+1}+a_{0} \cdot E_{t}=0 .
$$


Multiplying both sides by $c^{\mathrm{T}}$ and taking into account the formula (1), we conclude that for each trend $T_{t}$ from the family $C$, we have the following equality:

$$
a_{n} \cdot T_{t+n}+a_{n-1} \cdot T_{t+(n-1)}+\ldots+a_{1} \cdot T_{t+1}+a_{0} \cdot T_{t}=0 .
$$

Final result: how to reach stationarity. If we now apply the same linear operator to the signal $x_{t}=T_{t}+f_{t}$, then, due to (14), the effect of the trend disappears, and thus, only the $f$-result remains:

$$
\begin{gathered}
a_{n} \cdot x_{t+n}+a_{n-1} \cdot x_{t+(n-1)}+\ldots+a_{1} \cdot x_{t+1}+a_{0} \cdot x_{t}= \\
a_{n} \cdot f_{t+n}+a_{n-1} \cdot f_{t+(n-1)}+\ldots+a_{1} \cdot f_{t+1}+a_{0} \cdot f_{t} .
\end{gathered}
$$

Since the process $f_{t}$ is stationary, the right-hand side of the formula (15) is also stationary.

Thus, for each process, by considering an appropriate linear combination of this process $x_{t}$ and its shifts $x_{t+1}, x_{t+2}$, etc., we can get a stationary process. So, to be able to apply stationary-based techniques, we must find the values $a_{i}$ for which the linear combination

$$
a_{n} \cdot x_{t+n}+a_{n-1} \cdot x_{t+(n-1)}+\ldots+a_{1} \cdot x_{t+1}+a_{0} \cdot x_{t}
$$

is stationary.

How can we find such coefficients? To find the corresponding coefficients, we can use well-developed co-integration techniques (see, e.g., [3]) or, better yet, the newly developed techniques of stationary subspace analysis (see, e.g., [4] and references therein). These techniques find stationary lineae combinations of non-stationary processes. In our case, we need to apply this technique to the original series $x_{t}$ and to the time-shifted series $x_{t+1}, x_{t+2}$, etc.

\section{Acknowledgments}

We acknowledge the partial support of the Center of Excellence in Econometrics, Faculty of Economics, Chiang Mai University, Thailand. This work was also supported in part by the National Science Foundation grant HRD-1242122 (CyberShARE Center of Excellence).

One of the authors (VK) is thankful to Mohsen Pourahmadi for valuable discussions. 


\section{References}

1. R. F. Engle and C. W. J. Granger, "Co-integration and error orrection: representation, estimation, and testing", Econometrica, 1987, Vol. 55, No. 2, pp. 251-276.

2. J. E. Gentle, Matrix Algebra: Theory, Computations, and Applications in Statistics, Springer Verlag, New York, 2007.

3. K. Neusser, Time Series Econometrics, Springer Verlag, Cham, Switzerland, 2016.

4. R. R. Sundararajan and M. Pourahmadi, "Stationary Subspace Analysis of nonstationary processes", Journal of Time Series Analysis, to appear. 